\title{
Flavour anomalies in rare decays at $\mathrm{LHCb}$
}

\section{Lorenzo Capriotti*†}

Alma Mater Studiorum Università di Bologna

INFN - Sezione di Bologna

E-mail: lorenzo.capriotti@unibo.it

Rare decays are powerful probes for Physics beyond the Standard Model (SM), as new particles can have a large impact on physics observables. Recent results on lepton universality tests and measurements of branching fractions and angular distributions of rare $b \rightarrow$ sll decays have shown tensions with the SM predictions. The $\mathrm{LHCb}$ experiment is ideally suited for the study of the these flavour anomalies, due to its large acceptance, precise vertexing and powerful particle identification capabilities. The latest results from $\mathrm{LHCb}$ on the flavour anomalies will be presented and their interpretation will be discussed.

The 39th International Conference on High Energy Physics (ICHEP2018)

4-11 July, 2018

Seoul, Korea

* Speaker.

${ }^{\dagger}$ On behalf of the LHCb Collaboration. 


\section{Introduction}

The phenomenology of electroweak interactions is well described in the Standard Model (SM) of particle physics by a Yang-Mills theory applied to the symmetry group $S U(2) \otimes U(1)$. Under this assumption, the requirement of gauge invariance of the SM Lagrangian implies that the lepton couplings do not depend on the lepton family; therefore, any difference in the decay amplitudes resides in the different volumes of the phase space, i.e. on the masses of the leptons. However, direct and indirect tests of lepton universality for $b \rightarrow \operatorname{sll}$ and $b \rightarrow c l v$ decays, measured by several experiments, seem to show that the electroweak interactions might be non-universal. In the SM, $b \rightarrow$ sll transition can only occur via loops, with $\mathscr{B} \sim 10^{-6}-10^{-7}$, and this makes them highly sensitive to contributions beyond the SM. These decays are described with a model-independent approach, using an effective operator-product-expansion, so that the Lagrangian is defined as a sum of current-current operators multiplied by complex coefficients $C_{i}$ (Wilson coefficients). This gives sensitivity in angular distributions and differential branching fractions, as different regions of the $q^{2}$ spectrum are described by different operators and their interference. In this work, the latest measurement performed by the LHCb esperiment on the anomalies in rare $b \rightarrow$ sll transitions, along with direct tests of lepton universality, are presented.

\section{Angular analysis of $b \rightarrow$ sll decays}

The LHCb experiment has performed an angular analysis of the decay $B^{0} \rightarrow K^{* 0} \mu^{+} \mu^{-}$, using $3 \mathrm{fb}^{-1}$ of data collected during 2011 and 2012 at a centre-of-mass energy of 7 and $8 \mathrm{TeV}$ (Run 1) [1]. A set of angular observables, for which the hadronic form factor uncertainties cancel at leading order, can be defined from the coefficients of the angular distribution [2]. Amongst them, the distribution of $P_{5}^{\prime}$ as a function of $q^{2}$ shows local deviations with respect to the SM predictions, with a global significance of $3.4 \sigma$. The LHCb experiment has also performed an angular analysis of the decay $\Lambda_{b}^{0} \rightarrow \Lambda^{0} \mu^{+} \mu^{-}$, using the full Run 1 statistics, plus $2 \mathrm{fb}^{-1}$ of data collected during 2015 and 2016 (during Run 2) [3]. All angular observables measured are compatible with SM predictions within $2.6 \sigma$. Figure 1 shows the distribution of $P_{5}^{\prime}$, along with results from ATLAS [4], Belle [5] and CMS [6], which are found to be compatible, and the measurements of the angular observables from $\Lambda_{b}^{0} \rightarrow \Lambda^{0} \mu^{+} \mu^{-}$decays.
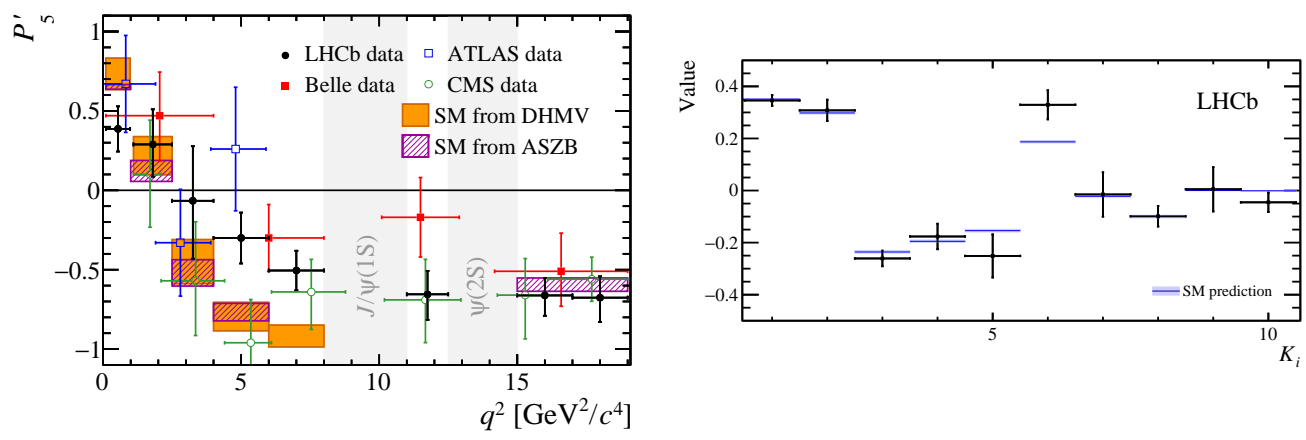

Figure 1: Distribution of $P_{5}^{\prime}$ from $B^{0} \rightarrow K^{* 0} \mu^{+} \mu^{-}$decays, measured by LHCb, ATLAS, Belle and CMS, with SM predictions (left); angular observables of the decay $\Lambda_{b}^{0} \rightarrow \Lambda^{0} \mu^{+} \mu^{-}$with SM predictions (right). 


\section{Differential branching fractions}

The LHCb experiment has measured the differential branching fractions, as functions of $q^{2}$, of several $b \rightarrow$ sll decays $[7,8,9,10,11]: B^{0} \rightarrow K^{0} \mu^{+} \mu^{-}, B^{+} \rightarrow K^{+} \mu^{+} \mu^{-}, B^{+} \rightarrow K^{*+} \mu^{+} \mu^{-}, B^{0} \rightarrow$ $K^{* 0} \mu^{+} \mu^{-}, B_{s}^{0} \rightarrow \phi \mu^{+} \mu^{-}$and $\Lambda_{b}^{0} \rightarrow \Lambda \mu^{+} \mu^{-}$. All these measurements are made using the full Run 1 dataset. As in the angular analysis of the decay $B^{0} \rightarrow K^{* 0} \mu^{+} \mu^{-}$, the regions corresponding to the $J / \psi$ and $\Psi(2 S)$ are vetoed to remove long distance effects. The differential branching fractions are shown in Figure 2 along with the theoretical predictions. It is evident that in the region below the charmonium resonances the measured branching fractions are consistently lower than the predicted values. The largest local discrepancy is $3.3 \sigma$, for the decay $B_{s}^{0} \rightarrow \phi \mu^{+} \mu^{-}$.
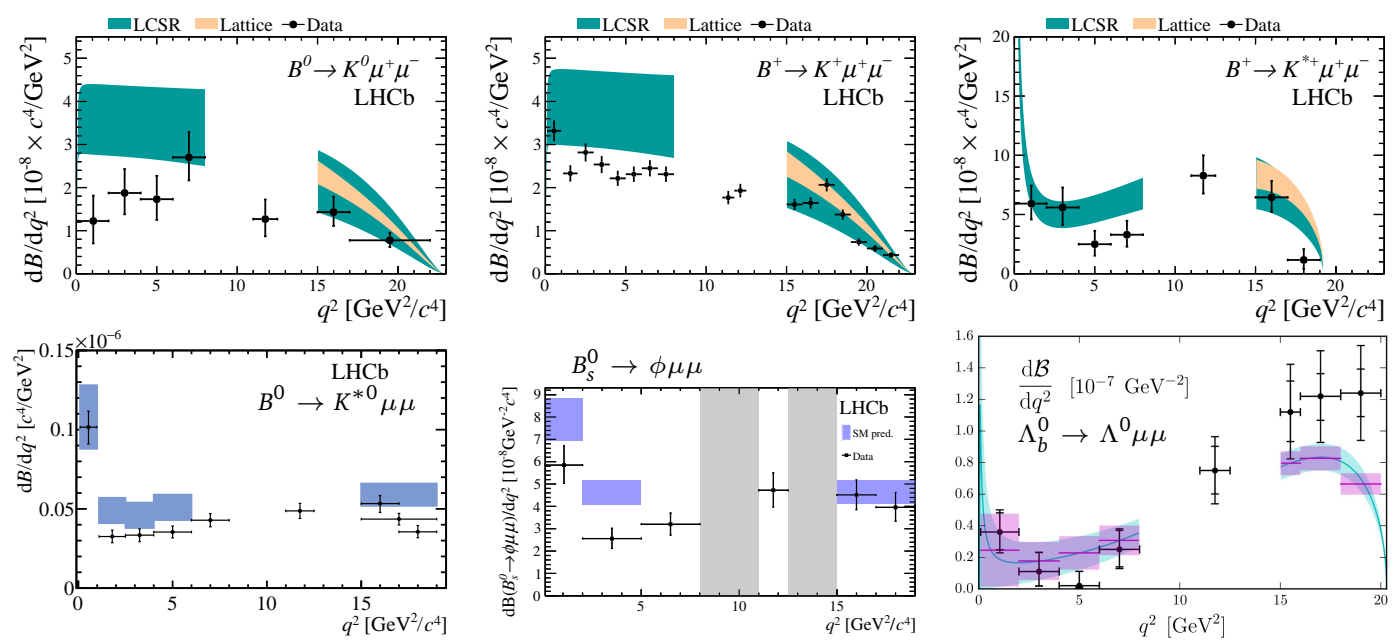

Figure 2: Differential branching fractions, as a function of $q^{2}$, of the decays measured by LHCb. The SM predictions are superimposed.

\section{Global fit from $b \rightarrow s \mu \mu$ anomalies}

Measurements of differential branching fractions and angular observables can be used as inputs to a global fit to the Wilson coefficients of the semileptonic operators $C_{9}$ and $C_{10}$. This has been done by several groups, using different subsets of data and different theoretical assumptions $[12,13,14]$. The global fits to the real part of $C_{9}$ and $C_{10}$, in all cases, points towards a shift in $C_{9}$ which can be as high as $5 \sigma$, depending on the model used for the fit. This would suggest that an additional contribution with respect to the SM is necessary to accommodate the data.

\section{Direct tests of lepton universality in $b \rightarrow$ sll decays}

Two direct tests of lepton universality have been performed by the LHCb experiment, in the decay channels $B^{+} \rightarrow K^{+} l^{-} l^{-}$and $B^{0} \rightarrow K^{* 0} l^{+} l^{-}$(where $l=e, \mu$ ) using the branching fractions double ratio as observable $[15,16]$. This is defined as

$$
R\left(K^{(*)}\right)=\frac{\mathscr{B}\left(B \rightarrow K^{(*)} \mu^{+} \mu^{-}\right)}{\mathscr{B}\left(B \rightarrow K^{(*)}\left(J / \psi \rightarrow \mu^{+} \mu^{-}\right)\right)} / \frac{\mathscr{B}\left(B \rightarrow K^{(*)} e^{+} e^{-}\right)}{\mathscr{B}\left(B \rightarrow K^{(*)}\left(J / \psi \rightarrow e^{+} e^{-}\right)\right)} .
$$


The double ratio reduces the experimental systematic uncertainties. The measured values are

$$
\begin{gathered}
R(K)=0.745_{-0.074}^{+0.090} \pm 0.036 \text { at central } q^{2} \in[1.0,6.0] \mathrm{GeV} / c^{2}(2.6 \sigma), \\
R\left(K^{*}\right)=0.66_{-0.07}^{+0.11} \pm 0.03 \text { at low } q^{2} \in[0.045,1.1] \mathrm{GeV} / c^{2}(2.1 \sigma-2.3 \sigma), \\
R\left(K^{*}\right)=0.69_{-0.07}^{+0.11} \pm 0.05 \text { at central } q^{2} \in[1.1,6.0] \mathrm{GeV} / c^{2}(2.4 \sigma-2.5 \sigma),
\end{gathered}
$$

where the deviation from the SM prediction is indicated in parentheses. All measurements fluctuate in the same direction, and they are consistent with a shift in the muonic $C_{9}$.

\section{References}

[1] R. Aaij et al. (LHCb coll.), Angular analysis of the $B^{0} \rightarrow K^{* 0} \mu^{+} \mu^{-}$decay using $3 \mathrm{fb}^{-1}$ of integrated luminosity, JHEP 02 (2016) 104 [hep-ex/1512.04442]

[2] S. Descotes-Genon, T. Hurth, J. Matias, J. Virto, Optimizing the basis of $B \rightarrow K^{*} l^{+} l^{-}$observables in the full kinematic range, JHEP 05 (2013) 137 [hep-ph/1305:137]

[3] R. Aaij et al. (LHCb coll.), Angular moments of the decay $\Lambda_{b}^{0} \rightarrow \Lambda \mu^{+} \mu^{-}$, hep-ex/1808.00264 (2018)

[4] M. Aaboud et al. (ATLAS coll.), Angular analysis of $B_{d}^{0} \rightarrow K^{*} \mu^{+} \mu^{-}$decays in pp collisions at $\sqrt{s}=8 \mathrm{TeV}$ with the ATLAS detector, hep-ex/1805.04000 (2018)

[5] S. Wehle et al. (Belle coll.), Lepton-Flavor-Dependent Angular Analysis of $B \rightarrow K^{*} l^{+} l^{-}$, PRL 118 (2017) 111801 [hep-ex/1612.05014]

[6] A.M. Sirunyan et al. (CMS coll.), Measurement of angular parameters from the decay $B^{0} \rightarrow K^{*} \mu^{+} \mu^{-}$ in proton-proton collisions at $\sqrt{s}=8 \mathrm{TeV}$, PLB 781 (2018) 517-541 [hep-ex/1710.02846]

[7] R. Aaij et al. (LHCb coll.), Differential branching fractions and isospin asymmetries of $B \rightarrow K^{(*)} \mu^{+} \mu^{-}$decays, JHEP 06 (2014) 133 [hep-ex/1403.8044]

[8] R. Aaij et al. (LHCb coll.), Measurements of the $S$-wave fraction in $B^{0} \rightarrow K^{+} \pi^{-} \mu^{+} \mu^{-}$decays and the $B \rightarrow K^{*}(892)^{0} \mu^{+} \mu^{-}$differential branching fraction, JHEP 11 (2016) 047 [hep-ex/1606.04731]

[9] R. Aaij et al. (LHCb coll.), Angular analysis and differential branching fraction of the decay $B_{s}^{0} \rightarrow \phi \mu^{+} \mu^{-}$, JHEP 09 (2015) 179 [hep-ex/1506.08777]

[10] R. Aaij et al. (LHCb coll.), Differential branching fraction and angular analysis of $\Lambda_{b}^{0} \rightarrow \Lambda \mu^{+} \mu^{-}$ decays, JHEP 06 (2015) 115 [hep-ex/1503.07138]

[11] W. Detmold, S. Meinel, $\Lambda_{b} \rightarrow \Lambda l^{+} l^{-}$form factors, differential branching fraction, and angular observables from lattice QCD with relativistic b quarks, PRD 93 (2016) 074501 [hep-lat/1602.01399]

[12] S. Descotes-Genon, L. Hofer, J. Matias, J. Virto, Global analysis of $b \rightarrow$ sll anomalies, JHEP 06 (2016) 092 [hep-ph/1510.04239]

[13] F. Mahmoudi, T. Hurt, S. Neshatpour, Present Status of $b \rightarrow$ sll anomalies, hep-ph/1611.05060 (2016)

[14] W. Altmannshofer, C. Niehoff, P. Stangl, D. M. Straub, Status of the $B \rightarrow K^{*} \mu^{+} \mu^{-}$anomaly after Moriond 2017, EJPC 77 (2017) 377 [hep-ph/1703.09189]

[15] R. Aaij et al. (LHCb coll.), Test of Lepton Universality Using $B^{+} \rightarrow K^{+} l^{+} l^{-}$Decays, PRL 113 (2014) 151601 [hep-ex/1406.6482]

[16] R. Aaij et al. (LHCb coll.), Test of lepton universality with $B^{0} \rightarrow K^{* 0} l^{+} l^{-}$decays, JHEP 08 (2017) 055 [hep-ex/1705.05802] 